egneuro,03(01):1-2,2021

\title{
Changing Paradigm for Brain AVM Treatment
}

\author{
KarunaTamrakar Karki ${ }^{1}$ \\ ${ }^{1}$ Department of Neurosurgery,B and C Medical College Teaching Hospital and Research Center, Birtamode, \\ Jhapa, Nepal \\ Correspondence: \\ Dr. KarunaTamrakarKarki \\ Department of Neurosurgery \\ B and C Medical College Teaching Hospital and Research Center, Birtamode, Jhapa, Nepal \\ Email: tamrakarkaruna@gmail.com
}

$\mathrm{B}$ rain AVMs are the most common vascular malformation with reported hemorrhagic risk of $4 \%$ per year with possibilities of neurological deficits and death are $<50 \%$ and $<10 \%$ respectively. ${ }^{1}$ The main purpose of brain AVM treatment is to reduce the hemorrhage risk which can be achieved by isolating the nidus from the circulation. Currently AVMs are managed by microsurgery, endovascular embolization and stereotactic radiosurgery (SRS) alone or in combination. Mainstay of the therapy has always been the microsurgery for low grade AVMs. However potential complications after microsurgery in eloquent area, deep venous drainage, high flow fistula and other additional angiographic features have led to less invasive therapeutic approaches. ${ }^{2}$

ARUBA (A Randomized trial of Unruptured Brain Arteriovenous malformation) study was a simple study justified only by longitudinal data on natural history of unruptured brain AVMs showing mix picture of primary outcome. There was patient selection bias and only low grade AVMs were included in the study with short follow-up period of 33.3 months. Controversy over the ARUBA trail had led to conflicting pattern of treatment. There was no subgroup analysis evaluating the outcome of different treatment modalities on different grades of brain AVM. The main hypothesis of this trial showed no differences exist between surgical and medical management. ${ }^{3}$ However, ARUBA trail was halted prematurely with several critics. Large number of ARUBA patients was treated with standalone embolization which was also a point of criticism.

\section{1}

Date submitted: 20/ 06/2021

Date accepted: 1/07/2021
Recent studies have evaluated endovascular embolization with non-adhesive liquid embolic material and gamma knife radiosurgery are useful methods of AVM treatment. ${ }^{4}$ Endovascular embolization is the best choice for transforming large volume AVMs which are not ideal for single session SRS into radio compatible size. Reductive embolization of large volume AVMs are associated with improved outcome after SRS. Embolization can also reduce high risk angiographic features such as perinidalintranidal aneurysms and intranidal high flow arteriovenous fistulas.

Some neuro interventionists considered three major fundamentals for AVM treatment depending upon the eloquent area, symptomatic prior to intervention and post intervention neurological deterioration. Modified Spletzer-Martin (SM) grade III has been classified into 3 subtypes based on size of nidus to accurately predict the surgical risk. ${ }^{5} \mathrm{SM}$ grade II and III treatment modalities have been changing particularly affected by development in SRS and endovascular embolization.

Inadequate AVM obliteration rates have been observed in embolized AVM treated with SRS. There are numerous proposals to explain the underlying mechanism of unsuccessful SRS among post embolized brain AVMs. ${ }^{6}$ Embolization may promote angiogenesis converting a relatively dormant nidus to a dynamic lesion with increased radio-resistance. Embolic material may absorb or scatter the radiation beams delivered during SRS, thus by reduces overall radiation dose to nidus. Another theory explains the possibility of angiogenesis induced inflammation and

egneuro Volume 03, Issue 01, 2021 
hemodynamic alteration causing difficulty in defining the boundaries of nidus. Embolization can also fragment the nidus into noncontiguous compartment transforming into diffuse one thus creating difficulty in lesion targeting during SRS. Inconsistent result may be caused by individuals AVM angioarchitecture with or without additional high risk angiographic features. Furthermore the effect of these high risk features on the natural history of AVM is not well defined.

Endovascular embolization for AVM improves the risk benefit profile of combined treatment with SRS has to be revisited. Non-adhesive liquid embolic material called ethylene vinyl alcohol (ONYX; EV3 USA), (SQUID; Balt Co) and more recently precipitating hydrophobic injectable liquid (PHIL; Microvention USA) present greater volume reduction with deeper penetrating quality into the nidus and carries less radio-surgical dose attenuation than the older embolic agents. ${ }^{7}$ Newly invented non adhesive embolic agents are ready to use products. They have optimal visibility during embolization and create non saturated radio-opaque cast thus facilitating staged embolization. These are much more compatible for surgical resection. They considerably produce fewer beam hardening artifacts on CT as compared to tantalum based embolic materials. Improvisation on embolic materials, micro catheters and micro guide wires additionally benefited to deliver embolic agents to the fragile vessel closer to AVM nidus. Improvements in diagnostic and therapeutic potential have allowed the neuro intervention to offer a steadily increasing quality in effective treatment of certain therapeutically challenging vascular malformations. Continuous advancement of techniques and parallel growth of knowledge and skills has taken neuro intervention into a and arterial access has made endovascular therapy more safe and curative.

Safety of AVM treatment depends on natural history, better understanding of device development and proper treatment selection. Further innovations are expected to change the treatment modalities. Curative or standalone embolization is safe and effective allowing complete obliteration of AVM with lower complication rates. ${ }^{8}$ Considering utility of combined therapy one should be always aware of patient's outcome as they constantly depend on their ability to perform a proper, safe and adequate embolization. Large Cohort study or Randomization controlled trial study has to be reconsidered the treatment strategy of both hemorrhagic and non-hemorrhagic onset of brain AVMs.

\section{References:}

1. Shaligram SS, Winkler E, Cooke D, Su H. Risk factors for hemorrhage of brain arteriovenous malformation. CNS NeurosciTher. 2019 Oct; 25(10):1085-1095. doi: 10.1111/cns.13200. Epub 2019 Jul 29.

2. Derdeyn CP, Zipfel GJ, Albuquerque FC, Cooke DL, Feldmann E, Sheehan JP, Torner JC; American Heart Association Stroke Council. Management of Brain Arteriovenous Malformations: A Scientific Statement for Healthcare Professionals From the American Heart Association/American Stroke Association. Stroke. 2017 Aug; 48(8):e200-e224.

3. Feghali J, Huang J. Updates in arteriovenous malformation management: the post-ARUBA era. Stroke Vasc Neurol. 2019 Sep 21; 5(1):34-39.

4. Mendes GAC, Kalani MYS, Iosif C, Lucena AF, Carvalho $\mathrm{R}$, Saleme S, Mounayer C. Transvenous Curative Embolization of Cerebral Arteriovenous Malformations: A Prospective Cohort Study. Neurosurgery. 2018 Nov 1; 83(5):957-964.

5. Komatsu K, Takagi Y, Ishii A, Kikuchi T, Yamao Y, Yoshida K, Miyamoto S. Changes in treatment strategy over time for arteriovenous malformation in a Japanese high-volume center. BMC Neurol. 2020 Nov 5; 20(1):404.

6. Russell D, Peck T, Ding D, Chen CJ, Taylor DG, Starke RM, Lee CC, Sheehan JP. Stereotactic radiosurgery alone or combined with embolization for brain arteriovenous malformations: a systematic review and meta-analysis. J Neurosurg. 2018 May; 128(5):1338-1348.

7. Lord J, Britton H, Spain SG, Lewis AL. Advancements in the development on new liquid embolic agents for use in therapeutic embolisation. J Mater Chem B. 2020 Sep 23; 8(36):8207-8218.

8. Iosif C, de Lucena AF, Abreu-Mattos LG, Ala VHE, ElGhanam A, Saleme S, Caire F, Mounayer C. Curative endovascular treatment for low-grade Spetzler-Martin brain arteriovenous malformations: a single-center prospective study. J Neurointerv Surg. 2019 Jul; 11(7):699-705. 